\title{
Nutritional Quality and Acceptability Evaluation of Ogi Flour Biofortified with Garlic and Ginger
}

\author{
Olaniran Abiola Folakemi ${ }^{1}$, Abiose Sumbo Henrietta ${ }^{2}$ and Gbadamosi Saka Olusunkami ${ }^{2}$ \\ 1. Department of Microbiology, Landmark University, Omu-Aran 251101, Nigeria \\ 2. Department of Food Science and Technology, Obafemi Awolowo University, Ile-Ife 220282, Nigeria
}

\begin{abstract}
Ogi an affordable complementary food is of paramount importance due to its popularity as diets of many native African populations. The study explored effects of biofortification with garlic and ginger on acceptability and nutritional quality of ogi flour prepared from sorghum and quality protein maize by natural fermentation technique. Pasting properties, bulk density, water absorption capacity, total reducing sugar and sensory characteristics were evaluated. Biofortified samples showed stable total reducing sugar content, increase in bulk density, decrease in water absorption capacity and peak time during storage. The pasting temperatures of biofortified ogi flour were below boiling temperature thus formed paste in hot water below the boiling point. Ogi (maize) with $2 \%$ garlic-2\% ginger and ogi (sorghum) with $4 \%$ garlic-2\% ginger were the most preferred with no significant difference $(p<0.05)$. Biofortification with garlic and ginger at 2 and $4 \%$ improved the quality attributes and acceptability of ogi flour. The study on biofortification of ogi with garlic and ginger is germane in formulation of nourishing weaning food that is light, less bulky for babies, nursing mothers and malnourished populations.
\end{abstract}

Key words: Biofortification, garlic, ginger, acceptability, nutritional, ogi flour.

\section{Introduction}

Maize is a major staple food consumed by societies with several food preferences and backgrounds in sub-Saharan Africa. It accounts for approximately one-fifth of the diets and source of protein consumed in West Africa [1]. Sorghum is ranked second after maize as most important cereal in Africa [2]. Quality protein maize (QPM) has higher amounts of protein (10.56\%), fat (3.56\%) and ash (1.72\%) more than regular maize $(10.44,2.66$ and $1.28 \%$, correspondingly) [3]. Sorghum is an important cereal grain food, grown globally and could be used as a substitute for conventional cereals due to its high bioactive compounds, minerals, dietary fiber, vitamin E and carotenoids content [4]. Sorghum is suitable as weaning food due to its protein digestibility that ranges from 36.4 to $74 \%$ upon wet cooking. Ogi cereal gruel is a staple food of several communities in

Corresponding author: Olaniran Abiola Folakemi, Ph.D., research fields: food science and technology.
Nigeria which can be produced from maize, sorghum and millet [5]. Ogi from locally available cereals remains the most important complementary foods in West African countries especially in Nigeria, Benin, Ghana and Senegal. It is easy-to-get and cheap when compared with canned baby food [6]. Garlic (Allium sativum) and ginger (Zingiber officinale) are rich in proteins, phosphorous, potassium, calcium, magnesium and carbohydrates [7]. Biofortified food crops involve combination of high yielding and high nutrient crop varieties. Commercial fortification is addition of essential nutrients at the time of food processing to increase the content of essential micronutrients in food [8]. Garlic has functional antimicrobial, anti-carcinogenic, antihypertensive effects, hepato and cardio protection properties. In spite of the various health benefits of garlic; overall consumption of raw garlic is declining because of its pungent smell and taste [9]. They are universally acceptable; relatively inexpensive and well tolerated by most people, making it an indispensable ingredient 
of food processing throughout the world [7]. Ogi has high moisture content, which predisposes it to spoilage hence reduction of moisture content by drying can elongate their shelf-life, makes it handier and allows reconstitution of the ogi powder with ease. The consistency and thickness of complementary food given to the children determines its acceptability. Hence, this study explored the influence of biofortification of garlic and ginger on the quality of ogi flour in terms of the bulk density, water absorption capacity, pasting properties and its overall acceptability during storage.

\section{Material and Methods}

\subsection{Powdered Garlic and Ginger Preparation}

Freshly harvested garlic bulbs and ginger rhizomes (white variety) were washed; skinned and weighed before drying using hot air oven (Gallenkamp, UK) at $65{ }^{\circ} \mathrm{C}$ for $12 \mathrm{~h}$. It was then milled in a grinder (Marlex Appliances PVT, Mumbai, India), sieved (50 to $60 \mu \mathrm{m}$ mesh size) and packaged [7].

\subsection{Ogi Preparation Biofortified with Garlic and Ginger}

White Quality protein maize
(ART/98/SW06/OB/W) was obtained from the Institute of Agricultural Research and Training (I.A.R.T.), Ibadan, Nigeria. Sorghum (red variety) was purchased from a local market in Ile-Ife, Osun State, Nigeria. The grains were sorted, weighed $(15 \mathrm{~kg})$ each and steeped individually for $72 \mathrm{~h}$. The grains were drained, wet-milled to smooth paste using an attrition mill without sieving. The smooth pastes $(1 \mathrm{~kg})$ were weighed into 8 portions and $500 \mathrm{~mL}$ of distilled water was added to each to form slurry. Garlic and ginger powder were added for biofortification and labeled as follows: A: control samples (without garlic/ginger); B: Ogi + 2\% Garlic; C: Ogi + 4\% Garlic; D: Ogi $+2 \%$ Ginger; E: Ogi + 4\% Ginger; F: Ogi + 2\% Garlic-2\% Ginger; G: Ogi +2\% Garlic-4\% Ginger; H: Ogi + 4\% Garlic-2\% Ginger. The slurry was evenly homogenized and fermented for $24 \mathrm{~h}$ at ambient temperature $\left(27 \pm 2{ }^{\circ} \mathrm{C}\right)$. The fermented slurry was then decanted; pressed inside a muslin cloth bag to squeeze out excess water to form cake [10].

\subsection{Preparation of Biofortified Ogi Flour}

Ogi flour was obtained by drying the ogi cake using cabinet dryer at $42 \pm 2{ }^{\circ} \mathrm{C}$ for $48 \mathrm{~h}$. The dried cake was dry milled to aperture of $50 \mu \mathrm{m}$. The flour was cooled to room temperature before packaging into polyethylene bag and sealed with an electric sealer. The packaged samples were stored at ambient temperature for 16 weeks during which samples were obtained for analysis.

\subsection{Determination of Total Reducing Sugars}

The total reducing sugar content was determined using the Dinitrosalicyclic acid (DNSA) reagent method as described by Adeniran and Abiose [11]. One (1) millilitre of ethanolic extract was dispensed into test tubes and $2 \mathrm{~mL}$ of DNSA was added. The mixture was boiled for 5 min then rapidly cooled under running water; $7 \mathrm{~mL}$ of distilled water was added. The absorbance of the mixture was read at 540 $\mathrm{nm}$ in a UV Spectrophotometer against reagent blank. Calibration curve was prepared with different concentrations of glucose.

\subsection{Determination of Total Free Amino Acid}

Ninhydrin colorimetric method as described by Omafuvbe [12] was used. To $1 \mathrm{~mL}$ of the aliquot in a properly labeled test tube, $0.5 \mathrm{~mL}$ of cyanide acetate buffer (pH 5.4) and $0.5 \mathrm{~mL}$ of $3.0 \%$ ninhydrin solution in 2-methoxyethanol were added. The tubes were heated in a boiling water bath for 15 min after which $10 \mathrm{~mL}$ of isopropyl-alcohol: water mixture (1:1) was added rapidly and the solution was cooled to room temperature. The optical density (OD) of the solution was read at $570 \mathrm{~nm}$ in a spectrophotometer (Model SP9, PyeUnican UK). The concentration of free amino acids in the sample was extrapolated from a standard curve of known concentrations of glycine. 


\subsection{Functional Properties Determination}

Functional properties such as bulk density, water absorption capacity using [13] method and pasting properties of ogi flour with and without ginger and/or garlic were determined.

\subsection{Bulk Density}

Ogi flour samples (50 g) were weighed into a 100 $\mathrm{mL}$ measuring cylinder, empty and filled space of the measuring cylinders occupied by the sample was recorded. Cylinders were tapped until constant volume was attained. Bulk density $(\mathrm{g} / \mathrm{mL})$ was then calculated using the equation below with an average value of 10 measurements.

$$
\text { Bulk density }=\frac{\text { weight of flour }(g)}{\text { volume of flour }(m L)}
$$

\subsection{Water Absorption Capacity (WAC)}

The WAC of the ogi flour was determined at room temperature. Ogi flour (2 g) was weighed separately into a pre-weighed centrifuge tube and $20 \mathrm{~mL}$ of distilled water was dispensed into the sample. The contents were mixed for $30 \mathrm{~s}$ every 5 min using a glass rod. After $10 \mathrm{~min}$, it was centrifuged at 1,788 rpm for $20 \mathrm{~min}$. The supernatant was cautiously decanted, drained for $10 \mathrm{~min}$ and weighed. The water absorption capacity was expressed as percentage of the volume of water absorbed by the weight of the sample.

$$
\text { WAC }=\frac{\text { Volume of water absorbed }(\mathrm{mL})}{\text { weig ht of the sample }(\mathrm{g})} \times 100(\%)
$$

\subsection{Determination of Pasting Properties}

Pasting properties was determined using Rapid Visco Analyzer (RVA). Ogi flour (3 g) was weighed; $5 \mathrm{~mL}$ of distilled water was dispensed into test canister. The paddle was placed into the canister and the blade vigorously jogged through the slurry as the test proceeded and terminated automatically. The slurry was heated from 50 to $95{ }^{\circ} \mathrm{C}$ and cooled back to $50{ }^{\circ} \mathrm{C}$ within $12 \mathrm{~min}$ rotating the can at $160 \mathrm{rpm}$ through constant stirring of the content by a plastic paddle. Peak viscosity, setback viscosity, final viscosity, pasting temperature, pasting time, trough and breakdown value was estimated [14].

\subsection{Sensory Evaluation}

The sensory evaluation of reconstituted ogi flour was carried out using 9-point Hedonic scale. Panelist used to evaluate the ogi porridge was semi-trained but comprised of those acquainted with ogi porridge quality. Selection was based on interest and availability. Ogi porridge was prepared by reconstituting $10 \mathrm{~g}$ ogi flour with $50 \mathrm{~mL}$ of portable water; boiling water was added until gelatinization occurs. It was randomly served hot in coded plates and was rated based on colour, appearance, aroma, texture, taste and overall acceptability, where 9 represented like extremely and 1 dislike extremely. The data obtained for all the parameters were reported as means of 15 judgments [15].

\subsection{Statistical Analysis}

The mean value and standard deviation for all ogi flour samples were calculated in each case as entire determinations carried out were carried out in triplicates. ANOVA was done and the separation of the mean values uses Duncan's Multiple Range Test at $p$ for Social Scientists (SPSS) software, 16.0 version.

\section{Results}

Gradual decrease in total reducing sugar from 23.05-18.45 mg/mL (maize) and 22.46 to $18.80 \mathrm{mg} / \mathrm{mL}$ (sorghum) were observed in samples without garlic and ginger during 16 weeks of storage as presented in Figs. 1 and 2. It was observed that the total reducing sugar contents of flour samples containing $2 \%$ garlic gradually decreased from 22.32 to $20.77 \mathrm{mg} / \mathrm{mL}$ (maize) and from 22.50 to $21.53 \mathrm{mg} / \mathrm{mL}$ (sorghum) for the first 4 weeks and then became relatively stable till the end of storage. The total reducing sugar of ogi 


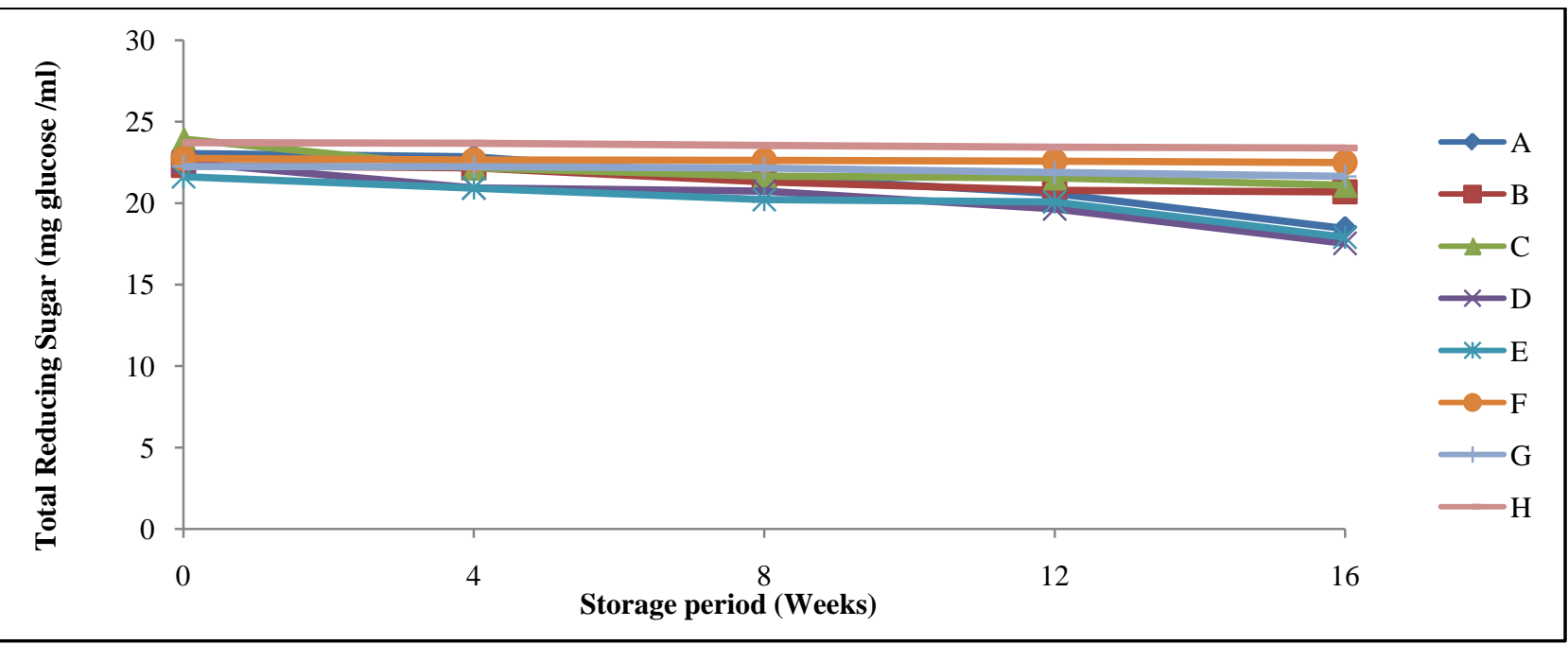

Fig. 1 Total reducing sugar of Ogi (maize) flour biofortified with garlic and ginger.

Sample codes: A: Ogi, B: Ogi + 2\% Garlic, C: Ogi + 4\% Garlic, D: Ogi + 2\% Ginger, E: Ogi + 4\% Ginger, F: Ogi + 2\% Garlic-2\% Ginger, G: Ogi + 2\% Garlic-4\% Ginger, H: Ogi + 4\% Garlic-2\% Ginger.

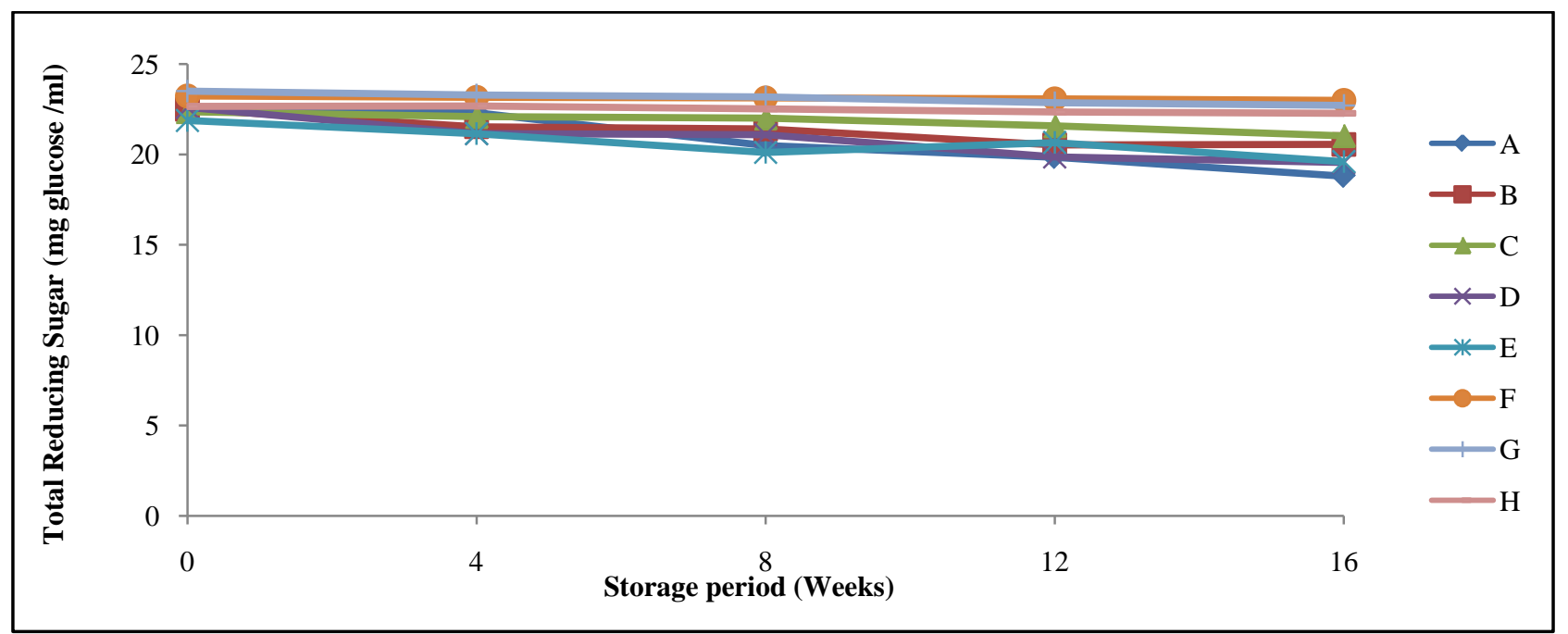

Fig. 2 Total reducing sugar of Ogi (sorghum) flour biofortified with garlic and ginger.

Sample codes: A: Ogi, B: Ogi + 2\% Garlic, C: Ogi + 4\% Garlic, D: Ogi + 2\% Ginger, E: Ogi + 4\% Ginger, F: Ogi +2\% Garlic-2\% Ginger, G: Ogi +2\% Garlic-4\% Ginger, H: Ogi +4\% Garlic-2\% Ginger.

flour (maize) with $4 \%$ garlic decreased from 23.94 to $21.66 \mathrm{mg} / \mathrm{mL}$ for 8 weeks and remained relatively stable till end of storage period. Relatively stable total reducing sugar content was observed in ogi flour (sorghum) with $4 \%$ garlic for 12 weeks followed by slight decrease for the remaining period of storage (20.66-19.60 $\mathrm{mg} / \mathrm{mL})$. The total reducing sugar contents of all ogi flour samples containing blends of $2 \%$ garlic- $2 \%$ ginger, $2 \%$ garlic- $4 \%$ ginger and $4 \%$ garlic-2\% ginger were stable during storage. The total free amino acid of ogi flour without biofortification, samples biofortified with $2 \%$ garlic, $4 \%$ garlic; $2 \%$ garlic-2\% ginger; $2 \%$ garlic-4\% ginger and $4 \%$ garlic-2\% ginger were stable throughout the 16 weeks of storage (Figs. 3 and 4). It was observed that ogi flour produced from sorghum without biofortification had the lowest total free amino acid content out of the samples throughout the period of storage. The bulk density of all ogi flour during storage ranged from 0.40 to $0.55 \mathrm{~g} / \mathrm{mL}$ (maize) and 0.50 to $0.67 \mathrm{~g} / \mathrm{mL}$ 


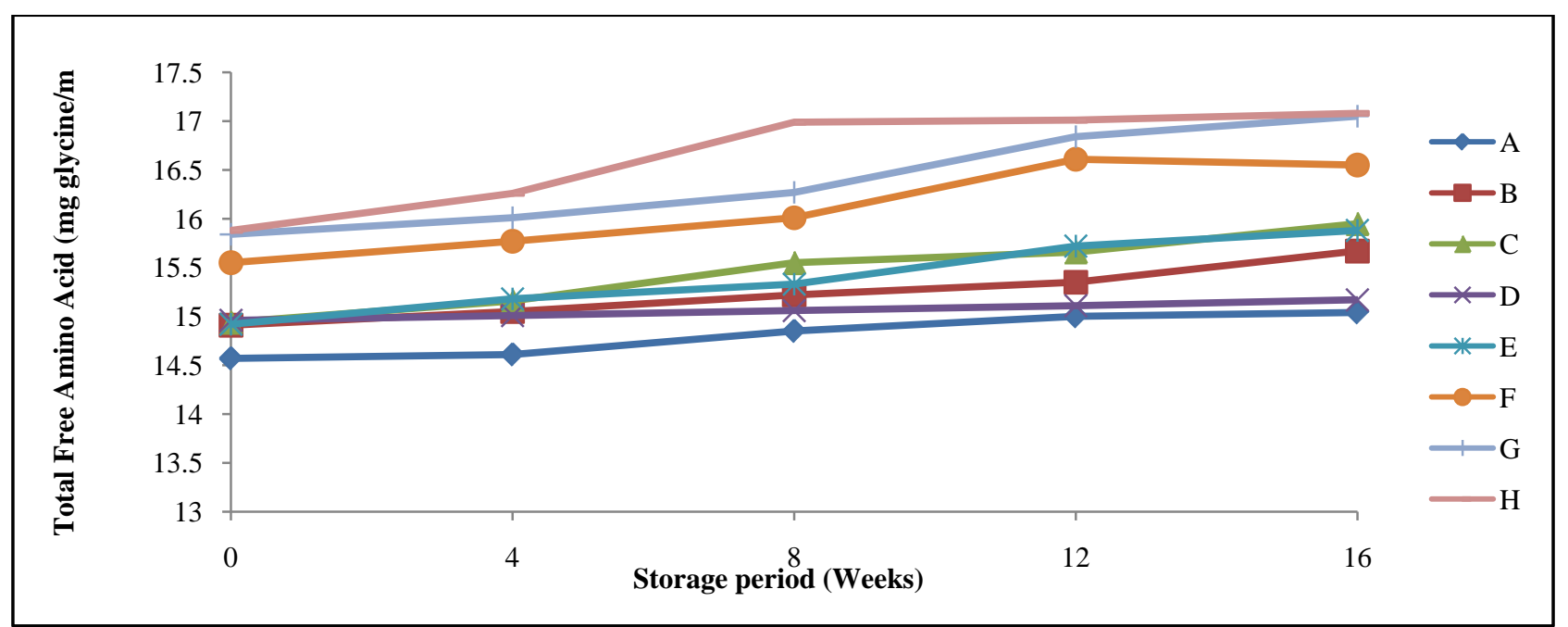

Fig. 3 Total free amino acid of $\mathrm{Ogi}$ (maize) flour biofortified with garlic and ginger.

Sample codes: A: Ogi, B: Ogi + 2\% Garlic, C: Ogi + 4\% Garlic, D: Ogi + 2\% Ginger, E: Ogi + 4\% Ginger, F: Ogi + 2\% Garlic-2\% Ginger, G: Ogi +2\% Garlic-4\% Ginger, H: Ogi + 4\% Garlic-2\% Ginger.

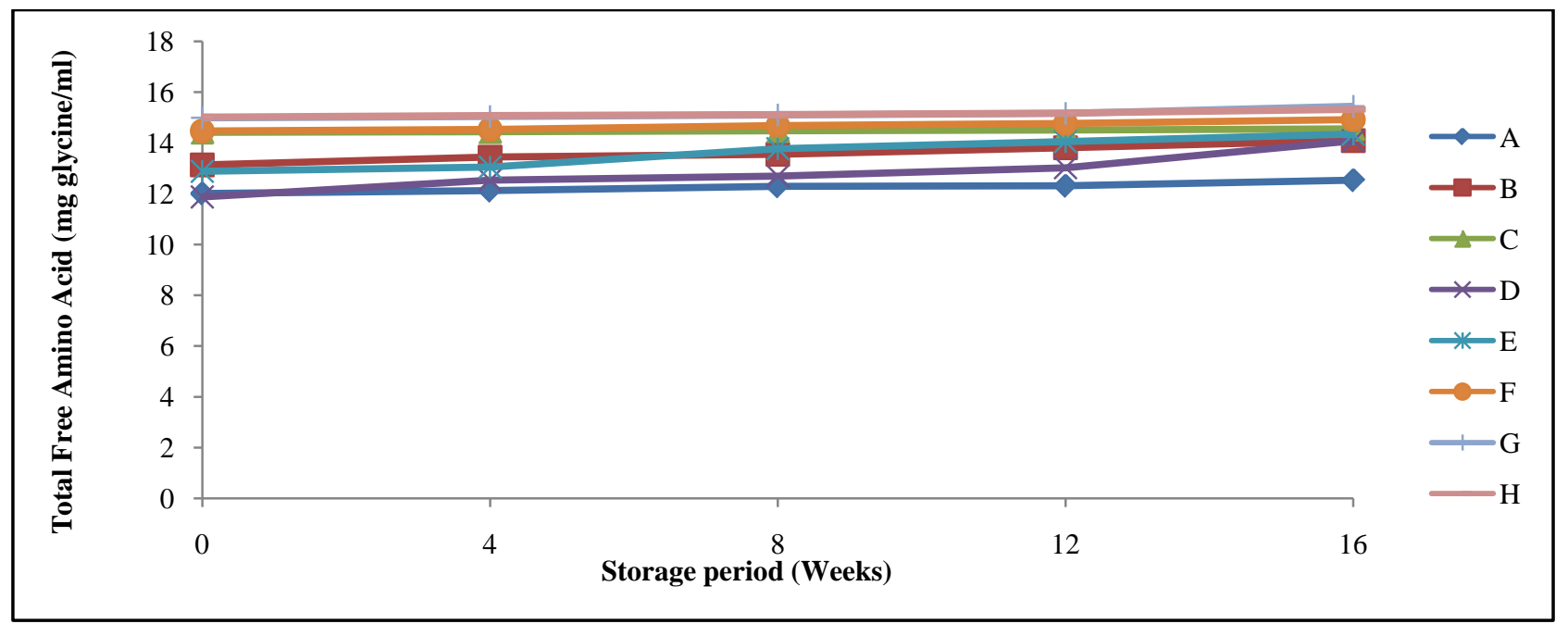

Fig. 4 Total free amino acid of $\mathrm{Ogi}$ (sorghum) flour biofortified with garlic and ginger.

Sample codes: A: Ogi, B: Ogi + 2\% Garlic, C: Ogi + 4\% Garlic, D: Ogi + 2\% Ginger, E: Ogi + 4\% Ginger, F: Ogi + 2\% Garlic-2\% Ginger, G: Ogi +2\% Garlic-4\% Ginger, H: Ogi + 4\% Garlic-2\% Ginger.

(sorghum) as presented in Table 1. It was observed that the bulk density of ogi flour produced from maize $(0.50 \mathrm{~g} / \mathrm{mL})$ was lower than sorghum's $(0.63 \mathrm{~g} / \mathrm{mL})$. The water absorption capacity (WAC) of all ogi flour samples ranged from 101.00 to $160.00 \%$ (maize) and 104.00 to $150.00 \%$ (sorghum). Biofortification of ogi flour with $2 \%$ garlic and $2 \%$ ginger singly increased the WAC (Table 1). However, decrease in water absorption capacity was observed in biofortified samples with $4 \%$ garlic, $4 \%$ ginger singly and samples containing combinations of garlic-ginger at different concentrations. The peak viscosity of ogi (maize) ranged from 119.67 to $222.08 \mathrm{RVU}$ and 161.17 to 301.08 RVU for ogi (sorghum) as presented in Tables 2 and 3.

Increase in the peak viscosity of most biofortified ogi flour may be due to availability of more starch granules with a high swelling capacity resulting in higher peak viscosity [16]. The addition of garlic-ginger to ogi flour increased the setback values (ability for formation of semi-solid gel/paste) which ranged from 55.58 to $92.58 \mathrm{RVU}$ for ogi (maize) and 
Table 1 Bulk density and water absorption capacities of Ogi flour biofortified with garlic and ginger.

\begin{tabular}{|c|c|c|c|c|}
\hline \multirow{2}{*}{ Sample/weeks } & \multicolumn{2}{|c|}{ Bulk density (g/mL) } & \multicolumn{2}{|c|}{ Water absorption capacity (\%) } \\
\hline & Maize & Sorghum & Maize & Sorghum \\
\hline $\mathrm{A} 0$ & $0.50 \pm 0.01^{\mathrm{b}}$ & $0.63 \pm 0.02^{\mathrm{b}}$ & $140.00 \pm 0.02$ & $130.00 \pm 0.06$ \\
\hline 16 & $0.49 \pm 0.02^{\mathrm{b}}$ & $0.60 \pm 0.03^{\mathrm{c}}$ & $101.00 \pm 0.05$ & $105.00 \pm 0.01$ \\
\hline B0 & $0.49 \pm 0.02^{\mathrm{b}}$ & $0.61 \pm 0.01^{\mathrm{c}}$ & $150.00 \pm 0.05$ & $140.00 \pm 0.02$ \\
\hline 16 & $0.48 \pm 0.02^{b}$ & $0.60 \pm 0.03^{c}$ & $145.00 \pm 0.01$ & $132.00 \pm 0.05$ \\
\hline $\mathrm{CO}$ & $0.48 \pm 0.01^{\mathrm{b}}$ & $0.60 \pm 0.04^{\mathrm{c}}$ & $130.00 \pm 0.08$ & $120.00 \pm 0.04$ \\
\hline 16 & $0.48 \pm 0.05^{\mathrm{b}}$ & $0.60 \pm 0.01^{\mathrm{c}}$ & $127.00 \pm 0.02$ & $115.00 \pm 0.09$ \\
\hline D0 & $0.55 \pm 0.01^{\mathrm{a}}$ & $0.67 \pm 0.02^{\mathrm{a}}$ & $160.00 \pm 0.03$ & $150.00 \pm 0.01$ \\
\hline 16 & $0.51 \pm 0.01^{\mathrm{b}}$ & $0.66 \pm 0.01^{\mathrm{a}}$ & $156.00 \pm 0.01$ & $149.00 \pm 0.08$ \\
\hline E0 & $0.52 \pm 0.03^{\mathrm{a}}$ & $0.65 \pm 0.03^{\mathrm{a}}$ & $120.00 \pm 0.02$ & $125.00 \pm 0.02$ \\
\hline 16 & $0.49 \pm 0.01^{\mathrm{b}}$ & $0.63 \pm 0.01^{\mathrm{b}}$ & $117.00 \pm 0.03$ & $120.00 \pm 0.03$ \\
\hline F0 & $0.43 \pm 0.02^{\mathrm{c}}$ & $0.54 \pm 0.02^{\mathrm{d}}$ & $130.00 \pm 0.02$ & $120.00 \pm 0.07$ \\
\hline 16 & $0.43 \pm 0.05^{\mathrm{c}}$ & $0.54 \pm 0.01^{\mathrm{d}}$ & $124.00 \pm 0.03$ & $118.00 \pm 0.05$ \\
\hline G0 & $0.42 \pm 0.01^{\mathrm{c}}$ & $0.51 \pm 0.03^{\mathrm{e}}$ & $130.00 \pm 0.05$ & $120.00 \pm 0.02$ \\
\hline 16 & $0.42 \pm 0.04^{c}$ & $0.51 \pm 0.01^{\mathrm{e}}$ & $129.00 \pm 0.02$ & $117.00 \pm 0.05$ \\
\hline HO & $0.40 \pm 0.02^{c}$ & $0.50 \pm 0.01^{\mathrm{e}}$ & $130.00 \pm 0.07$ & $110.00 \pm 0.05$ \\
\hline 16 & $0.40 \pm 0.05^{c}$ & $0.50 \pm 0.01^{\mathrm{e}}$ & $123.00 \pm 0.07$ & $104.00 \pm 0.02$ \\
\hline
\end{tabular}

Sample codes: A: Ogi, B: Ogi + 2\% Garlic, C: Ogi + 4\% Garlic, D: Ogi + 2\% Ginger, E: Ogi + 4\% Ginger, F: Ogi + 2\% Garlic-2\% Ginger, G: Ogi + 2\% Garlic-4\% Ginger, H: Ogi + 4\% Garlic-2\% Ginger.

Table 2 Pasting properties of Ogi flour (maize) biofortified with garlic and ginger.

\begin{tabular}{|c|c|c|c|c|c|c|c|}
\hline Sample & $\begin{array}{l}\text { Peak } \\
\text { viscosity }\end{array}$ & Trough viscosity & $\begin{array}{l}\text { Breakdown } \\
\text { viscosity }\end{array}$ & $\begin{array}{l}\text { Final } \\
\text { viscosity }\end{array}$ & Setback & Peak time & $\begin{array}{l}\text { Pasting } \\
\text { temperature }\end{array}$ \\
\hline $\bar{A}$ & $157.33 \pm 0.01^{\mathrm{bc}}$ & $90.08 \pm 0.02^{c}$ & $67.25 \pm 0.01^{\mathrm{c}}$ & $123.58 \pm 0.03^{\mathrm{d}}$ & $33.50 \pm 0.01^{\mathrm{d}}$ & $5.68 \pm 0.02^{\mathrm{ab}}$ & $83.35 \pm 0.01^{\mathrm{a}}$ \\
\hline B & $221.42 \pm 0.02^{\mathrm{a}}$ & $157.00 \pm 0.01^{\mathrm{a}}$ & $64.42 \pm 0.03^{c}$ & $237.08 \pm 0.01^{\mathrm{a}}$ & $80.08 \pm 0.02^{\mathrm{ab}}$ & $6.35 \pm 0.01^{\mathrm{a}}$ & $82.25 \pm 0.01^{\mathrm{a}}$ \\
\hline C & $222.08 \pm 0.01^{\mathrm{a}}$ & $140.42 \pm 0.03^{\mathrm{ab}}$ & $81.67 \pm 0.01^{b}$ & $233.00 \pm 0.02^{\mathrm{a}}$ & $92.58 \pm 0.01^{\mathrm{a}}$ & $6.45 \pm 0.03^{\mathrm{a}}$ & $82.59 \pm 0.02^{\mathrm{a}}$ \\
\hline $\mathrm{D}$ & $213.83 \pm 0.03^{\mathrm{ab}}$ & $107.08 \pm 0.01^{\mathrm{b}}$ & $106.75 \pm 0.02^{\mathrm{a}}$ & $196.67 \pm 0.01^{\mathrm{b}}$ & $89.58 \pm 0.03^{\mathrm{a}}$ & $5.25 \pm 0.01^{\mathrm{b}}$ & $81.47 \pm 0.03^{\mathrm{ab}}$ \\
\hline $\mathrm{E}$ & $119.67 \pm 0.01^{\mathrm{d}}$ & $70.92 \pm 0.02^{\mathrm{d}}$ & $48.75 \pm 0.01^{\mathrm{d}}$ & $126.50 \pm 0.02^{\mathrm{d}}$ & $55.58 \pm 0.01^{c}$ & $5.78 \pm 0.02^{\mathrm{ab}}$ & $81.45 \pm 0.01^{\mathrm{ab}}$ \\
\hline $\mathrm{F}$ & $133.50 \pm 0.02^{c}$ & $85.08 \pm 0.01^{\mathrm{c}}$ & $48.42 \pm 0.03^{d}$ & $145.25 \pm 0.01^{\mathrm{c}}$ & $60.17 \pm 0.02^{b}$ & $5.58 \pm 0.01^{\mathrm{ab}}$ & $82.35 \pm 0.02^{\mathrm{a}}$ \\
\hline G & $169.42 \pm 0.03^{b}$ & $109.08 \pm 0.02^{\mathrm{b}}$ & $60.34 \pm 0.01^{\mathrm{c}}$ & $230.57 \pm 0.03^{\mathrm{a}}$ & $61.15 \pm 0.01^{\mathrm{b}}$ & $5.25 \pm 0.02^{\mathrm{b}}$ & $83.65 \pm 0.01^{\mathrm{a}}$ \\
\hline $\mathrm{H}$ & $194.25 \pm 0.01^{\mathrm{ab}}$ & $148.75 \pm 0.01^{\mathrm{ab}}$ & $45.50 \pm 0.02^{\mathrm{d}}$ & $237.58 \pm 0.01^{\mathrm{a}}$ & $88.83 \pm 0.02^{\mathrm{a}}$ & $6.33 \pm 0.01^{\mathrm{a}}$ & $82.47 \pm 0.02^{\mathrm{a}}$ \\
\hline
\end{tabular}

Values are means $(n=3) \pm$ standard deviation. Means followed by different superscripts are significantly different $(p<0.05)$ along column according to Duncan multiple range test. Sample codes: A: Ogi, B: Ogi + 2\% Garlic, C: Ogi + 4\% Garlic, D: Ogi + 2\% Ginger, E: Ogi + 4\% Ginger, F: Ogi + 2\% Garlic-2\% Ginger, G: Ogi +2\% Garlic-4\% Ginger, H: Ogi +4\% Garlic-2\% Ginger.

Table 3 Pastingproperties of Ogiflour (sorghum) biofortified with garlic and ginger.

\begin{tabular}{llllllll}
\hline Sample & Peak viscosity & Trough viscosity & $\begin{array}{l}\text { Breakdown } \\
\text { viscosity }\end{array}$ & Final viscosity & Setback & Peak time & $\begin{array}{l}\text { Pasting } \\
\text { temperature }\end{array}$ \\
\hline A & $191.17 \pm 0.01^{\mathrm{d}}$ & $156.00 \pm 0.03^{\mathrm{c}}$ & $35.17 \pm 0.02^{\mathrm{d}}$ & $224.33 \pm 0.01^{\mathrm{bc}}$ & $68.33 \pm 0.03^{\mathrm{c}}$ & $6.25 \pm 0.02^{\mathrm{a}}$ & $82.58 \pm 0.01^{\mathrm{a}}$ \\
$\mathrm{B}$ & $301.08 \pm 0.03^{\mathrm{a}}$ & $240.33 \pm 0.01^{\mathrm{a}}$ & $60.75 \pm 0.02^{\mathrm{c}}$ & $413.83 \pm 0.03^{\mathrm{a}}$ & $73.50 \pm 0.01^{\mathrm{c}}$ & $5.19 \pm 0.01^{\mathrm{c}}$ & $81.47 \pm 0.02^{\mathrm{a}}$ \\
$\mathrm{C}$ & $278.67 \pm 0.02^{\mathrm{ab}}$ & $199.83 \pm 0.01^{\mathrm{b}}$ & $78.84 \pm 0.01^{\mathrm{b}}$ & $391.58 \pm 0.02^{\mathrm{a}}$ & $91.75 \pm 0.02^{\mathrm{ab}}$ & $5.38 \pm 0.03^{\mathrm{b}}$ & $83.65 \pm 0.01^{\mathrm{a}}$ \\
$\mathrm{D}$ & $251.83 \pm 0.01^{\mathrm{b}}$ & $220.08 \pm 0.02^{\mathrm{ab}}$ & $31.75 \pm 0.03^{\mathrm{e}}$ & $309.25 \pm 0.01^{\mathrm{ab}}$ & $80.17 \pm 0.03^{\mathrm{b}}$ & $5.44 \pm 0.01^{\mathrm{b}}$ & $82.58 \pm 0.03^{\mathrm{a}}$ \\
$\mathrm{E}$ & $161.17 \pm 0.02^{\mathrm{e}}$ & $123.25 \pm 0.01^{\mathrm{d}}$ & $37.92 \pm 0.01^{\mathrm{d}}$ & $247.33 \pm 0.02^{\mathrm{b}}$ & $104.08 \pm 0.01^{\mathrm{a}}$ & $6.35 \pm 0.02^{\mathrm{a}}$ & $81.47 \pm 0.01^{\mathrm{a}}$ \\
$\mathrm{F}$ & $197.33 \pm 0.01^{\mathrm{d}}$ & $158.92 \pm 0.03^{\mathrm{c}}$ & $38.45 \pm 0.02^{\mathrm{d}}$ & $249.25 \pm 0.01^{\mathrm{b}}$ & $98.33 \pm 0.02^{\mathrm{a}}$ & $5.85 \pm 0.01^{\mathrm{ab}}$ & $82.58 \pm 0.02^{\mathrm{a}}$ \\
$\mathrm{G}$ & $197.33 \pm 0.03^{\mathrm{d}}$ & $124.17 \pm 0.01^{\mathrm{d}}$ & $38.42 \pm 0.03^{\mathrm{d}}$ & $157.92 \pm 0.03^{\mathrm{d}}$ & $90.33 \pm 0.01^{\mathrm{ab}}$ & $5.19 \pm 0.03^{\mathrm{c}}$ & $83.66 \pm 0.01^{\mathrm{a}}$ \\
$\mathrm{H}$ & $235.88 \pm 0.01^{\mathrm{c}}$ & $108.58 \pm 0.02^{\mathrm{e}}$ & $127.30 \pm 0.01^{\mathrm{a}}$ & $183.42 \pm 0.01^{\mathrm{c}}$ & $74.84 \pm 0.02^{\mathrm{c}}$ & $5.25 \pm 0.01^{\mathrm{c}}$ & $80.47 \pm 0.03^{\mathrm{a}}$ \\
\hline
\end{tabular}

Values are means $(n=3) \pm$ standard deviation. Means followed by different superscripts are significantly different $(p<0.05)$ along column according to Duncan multiple range test. Sample codes: A: Ogi, B: Ogi + 2\% Garlic, C: Ogi + 4\% Garlic, D: Ogi + 2\% Ginger, E: Ogi + 4\% Ginger, F: Ogi + 2\% Garlic-2\% Ginger, G: Ogi + 2\% Garlic-4\% Ginger, H: Ogi +4\% Garlic-2\% Ginger. 
Table 4 Sensory evaluation of Ogi flour (maize) biofortified with garlic and ginger.

\begin{tabular}{|c|c|c|c|c|c|c|}
\hline \multirow[b]{2}{*}{ Samples } & \multicolumn{6}{|c|}{ Attributes } \\
\hline & Colour & Appearance & Aroma & Taste & Texture & $\begin{array}{l}\text { Overall } \\
\text { acceptability }\end{array}$ \\
\hline $\mathrm{A}$ & $6.72 \pm 0.01^{\mathrm{b}}$ & $7.50 \pm 0.05^{\mathrm{a}}$ & $6.35 \pm 0.02^{\mathrm{a}}$ & $6.45 \pm 0.01^{\mathrm{a}}$ & $6.01 \pm 0.03^{c}$ & $6.77 \pm 0.04^{\mathrm{a}}$ \\
\hline $\mathrm{B}$ & $6.61 \pm 0.02^{\mathrm{b}}$ & $6.55 \pm 0.03^{c}$ & $5.51 \pm 0.01^{\mathrm{c}}$ & $5.02 \pm 0.03^{c}$ & $6.25 \pm 0.01^{b}$ & $5.86 \pm 0.02^{b}$ \\
\hline $\mathrm{C}$ & $7.31 \pm 0.01^{\mathrm{a}}$ & $7.11 \pm 0.02^{b}$ & $5.54 \pm 0.03^{c}$ & $4.89 \pm 0.05^{\mathrm{C}}$ & $6.48 \pm 0.02^{\mathrm{a}}$ & $6.07 \pm 0.01^{\mathrm{b}}$ \\
\hline $\mathrm{D}$ & $6.25 \pm 0.03^{c}$ & $6.53 \pm 0.01^{\mathrm{c}}$ & $6.21 \pm 0.04^{\mathrm{a}}$ & $5.05 \pm 0.02^{c}$ & $6.35 \pm 0.03^{\mathrm{a}}$ & $6.02 \pm 0.05^{\mathrm{b}}$ \\
\hline $\mathrm{E}$ & $5.05 \pm 0.01^{\mathrm{e}}$ & $5.02 \pm 0.03^{f}$ & $4.77 \pm 0.01^{\mathrm{d}}$ & $4.83 \pm 0.04^{c}$ & $4.61 \pm 0.01^{\mathrm{d}}$ & $4.88 \pm 0.01^{\mathrm{d}}$ \\
\hline $\mathrm{F}$ & $6.27 \pm 0.04^{\mathrm{c}}$ & $6.19 \pm 0.01^{\mathrm{d}}$ & $6.05 \pm 0.02^{\mathrm{b}}$ & $6.25 \pm 0.01^{\mathrm{a}}$ & $6.39 \pm 0.05^{\mathrm{a}}$ & $6.74 \pm 0.03^{\mathrm{a}}$ \\
\hline G & $5.81 \pm 0.02^{\mathrm{d}}$ & $6.01 \pm 0.04^{\mathrm{e}}$ & $6.28 \pm 0.01^{\mathrm{a}}$ & $6.08 \pm 0.03^{b}$ & $6.15 \pm 0.01^{b}$ & $5.72 \pm 0.02^{c}$ \\
\hline $\mathrm{H}$ & $7.54 \pm 0.05^{\mathrm{a}}$ & $7.34 \pm 0.01^{\mathrm{a}}$ & $6.02 \pm 0.03^{b}$ & $4.96 \pm 0.02^{c}$ & $5.09 \pm 0.04^{\mathrm{d}}$ & $4.51 \pm 0.01^{\mathrm{d}}$ \\
\hline
\end{tabular}

Values are means $(n=15) \pm$ standard deviation. Means followed by different superscripts are significantly different $(p<0.05)$ along column according to Duncan multiple range test. Sample codes: A: Ogi, B: $O g i+2 \%$ Garlic, C: $O g i+4 \%$ Garlic, D: $O g i+2 \%$ Ginger, E: Ogi + 4\% Ginger, F: Ogi + 2\% Garlic-2\% Ginger, G: Ogi + 2\% Garlic-4\% Ginger, H: Ogi +4\% Garlic-2\% Ginger.

Table 5 Sensory evaluation of Ogi flour (sorghum) biofortified with garlic and ginger.

\begin{tabular}{|c|c|c|c|c|c|c|}
\hline \multirow[b]{2}{*}{ Samples } & \multicolumn{6}{|c|}{ Attributes } \\
\hline & Colour & Appearance & Aroma & Taste & Texture & $\begin{array}{l}\text { Overall } \\
\text { acceptability }\end{array}$ \\
\hline $\bar{A}$ & $6.74 \pm 0.02^{\mathrm{b}}$ & $6.55 \pm 0.01^{b}$ & $6.69 \pm 0.03^{a}$ & $6.28 \pm 0.01^{\mathrm{a}}$ & $6.92 \pm 0.04^{\mathrm{a}}$ & $7.05 \pm 0.01^{\mathrm{a}}$ \\
\hline B & $6.95 \pm 0.01^{\mathrm{a}}$ & $6.55 \pm 0.03^{b}$ & $6.51 \pm 0.02^{b}$ & $5.88 \pm 0.04^{b}$ & $6.31 \pm 0.02^{b}$ & $6.48 \pm 0.03^{c}$ \\
\hline $\mathrm{C}$ & $7.05 \pm 0.01^{\mathrm{a}}$ & $6.55 \pm 0.02^{b}$ & $5.32 \pm 0.01^{\mathrm{d}}$ & $5.51 \pm 0.03^{d}$ & $5.71 \pm 0.01^{\mathrm{d}}$ & $5.73 \pm 0.01^{\mathrm{d}}$ \\
\hline $\mathrm{D}$ & $6.41 \pm 0.03^{c}$ & $6.55 \pm 0.01^{\mathrm{b}}$ & $6.65 \pm 0.02^{\mathrm{a}}$ & $5.48 \pm 0.05^{\mathrm{d}}$ & $6.05 \pm 0.03^{c}$ & $6.44 \pm 0.02^{c}$ \\
\hline $\mathrm{E}$ & $6.33 \pm 0.01^{\mathrm{c}}$ & $5.82 \pm 0.04^{c}$ & $6.59 \pm 0.01^{b}$ & $6.12 \pm 0.02^{b}$ & $5.95 \pm 0.01^{\mathrm{c}}$ & $6.46 \pm 0.03^{c}$ \\
\hline $\mathrm{F}$ & $6.55 \pm 0.02^{c}$ & $7.01 \pm 0.01^{\mathrm{a}}$ & $5.54 \pm 0.03^{c}$ & $6.02 \pm 0.01^{\mathrm{b}}$ & $6.02 \pm 0.02^{C}$ & $6.73 \pm 0.04^{b}$ \\
\hline G & $6.48 \pm 0.04^{c}$ & $6.45 \pm 0.01^{\mathrm{b}}$ & $6.41 \pm 0.03^{b}$ & $5.73 \pm 0.01^{\mathrm{c}}$ & $5.68 \pm 0.01^{\mathrm{c}}$ & $6.11 \pm 0.02^{\mathrm{d}}$ \\
\hline $\mathrm{H}$ & $6.99 \pm 0.01^{\mathrm{a}}$ & $6.95 \pm 0.03^{\mathrm{a}}$ & $6.66 \pm 0.01^{\mathrm{a}}$ & $6.16 \pm 0.02^{\mathrm{a}}$ & $7.01 \pm 0.04^{\mathrm{a}}$ & $6.99 \pm 0.01^{\mathrm{a}}$ \\
\hline
\end{tabular}

Values are means $(n=15) \pm$ standard deviation. Means followed by different superscripts are significantly different $(p<0.05)$ along column according to Duncan multiple range test. Sample codes: A: Ogi, B: Ogi + 2\% Garlic, C: Ogi + 4\% Garlic, D: Ogi + 2\% Ginger, E: Ogi + 4\% Ginger, F: Ogi + 2\% Garlic-2\% Ginger, G: Ogi + 2\% Garlic-4\% Ginger, H: Ogi + 4\% Garlic-2\% Ginger.

ogi (sorghum) 68.33 to 104.08 RVU. The pasting temperature was between 81.45 and $83.65^{\circ} \mathrm{C}$ for ogi (maize) and 80.47 to $83.66{ }^{\circ} \mathrm{C}$ for ogi (sorghum). Biofortification with garlic improved the colour while ginger enhanced the aroma of ogi samples. Garlic-ginger samples were more preferred in terms of appearances and texture of ogi flour (Tables 4 and 5).

\section{Discussion}

Reducing sugar content in ogi has been reported as good attribute because methods of preparing weaning foods involve heat-processing [17]. Biofortification with garlic and ginger increased the total free amino acid ogi flour samples containing $2 \%$ garlic, $4 \%$ garlic; blends of $2 \%$ garlic- $2 \%$ ginger; $2 \%$ garlic- $4 \%$ ginger and $4 \%$ garlic- $2 \%$ ginger increased throughout the 16 weeks of storage. Similar observations have been reported during storage of cereal product [18]. Biofortification of ogi flour with garlic and garlic-ginger reduced the bulk density and was stable during storage. Samples of lower density are required for infants to allow them swallow with ease without choking. The low bulk density value corresponds to increase in compactness of flour particles; thereby increasing available energy content from ogi flour [19]. The reduction in water absorption observed in biofortified ogi flour with garlic and garlic-ginger is advantageous in formulation of healthy weaning foods that are low viscous and less bulky. The variance observed in WAC of flour samples may be due to molecular structure, particle size, and amylose/amylopectin ratio [20]. The pasting temperature 
of all biofortified the samples was lower than $100{ }^{\circ} \mathrm{C}$ as a result all samples formed paste in hot water below boiling point. The difference in the pasting characteristics of maize and sorghum flour could be attributed to their grain components [21]. Ogi (maize) with $2 \%$ garlic- $2 \%$ ginger and ogi (sorghum) with $4 \%$ garlic-2\% ginger were the most preferred in term of appearance, colour, aroma, taste, texture and overall acceptability out of all biofortified samples with no significant difference compared with control. Least preference observed for samples containing $4 \%$ garlic may be due to the strong flavour of garlic [22].

\section{Conclusions}

Results from this study showed that biofortification of ogi flour produced from maize or sorghum with garlic, ginger and garlic-ginger at 2 and $4 \%$ increased the guaranteed derivable nutrients and calories due to decrease in visousity and the bulkiness of the ogi flour. Their integration also improved its acceptability, as there was no significant difference $(p>0.05)$ in the overall acceptability between most preferred biofortified and the non-biofortified samples. Hence, these products are highly recommended as nourishing food for weaning, nursing mothers and malnourished population.

\section{Conflict of Interest Statement}

Authors declared no conflicting interests.

\section{Funding}

Not applicable.

\section{Authors' Contributions}

AFO and SHA conceptualized and designed the study; AFO performed the experiments, analyzed and interpreted the data. SOG analysed the pasting properties of the samples, SHA supervised and proof read the manuscript, and AFO was a major contributor in writing the manuscript. All authors read and approved the final manuscript.

\section{Acknowledgements}

I want to appreciate the Institute of Agricultural Research and Training (I.A.R.T.), Ibadan, Nigeria for providing quality protein maize used for this research.

\section{References}

[1] Macauley, H. 2015. “Cereal Crops: Rice, Maize, Millet, Sorghum, Wheat.” In Feed Africa an action plan African Agric Transform, 1-36.

[2] Stefoska-Needham, A., Beck, E. J., Johnson, S. K., and Tapsell, L. C. 2015. "Sorghum: An Underutilized Cereal Whole Grain with the Potential to Assist in the Prevention of Chronic Disease." Food Rev Int 31 (4): 401-37. Available from: http://www.tandfonline.com/doi/full/10.1080/87559129.2 015.1022832 .

[3] Ogunsakin, O. A., Banwo, K., Ogunremi, O. R., and Sanni, A. I. 2015. "Microbiological and Physicochemical Properties of Sourdough Bread from Sorghum Flour.” Int Food Res $J$.

[4] Anunciação, P. C., De Morais Cardoso, L., Vieira, J., Gomes, P., Mattos, C., Lucia, D., et al. 2017. "Comparing Sorghum and Wheat Whole Grain Breakfast Cereals: Sensorial Acceptance and Bioactive coMpound Content." Food Chem 221: 984-9. Available from: http://dx.doi.org/10.1016/j.foodchem.2016.11.065.

[5] Omemu, A. M., Okafor, U. I., Obadina, A. O., Bankole, M. O., and Adeyeye, S. A. O. 2018. "Microbiological Assessment of Maize Ogi Cofermented with Pigeon Pea." Food Sci Nutr 6 (5): 1238-53. Available from: http://doi.wiley.com/10.1002/fsn3.651.

[6] Opeifa, A. O., Olatidoye, O. P., Adesala, S. O., and Fayomi, M. J. 2015. "Production and Quality Evaluation of Ogi Produced from Fermented Maize and Horse Eye Bean (Mucuna urens).” Pakistan J Nutr 14 (7): 417-25. Available from: https://pdfs.semanticscholar.org/7946/36489492705832e 5da3fc431e876bcff3bb2.pdf.

[7] Olaniran, A. F., Abiose, S. H., and Adeniran, A. H. 2015. "Biopreservative Effect of Ginger (Zingiber officinale) and Garlic Powder (Allium sativum) on Tomato Paste.” J Food Saf. 35 (4): 440-52.

[8] Singla, P., and Grover, K. 2017. "Biofortified Cereal Crops: A Sustainable Approach for Food and Nutritional Security." Br J Appl Sci Technol Rev 24 (4): 1-13. Available from: http://www.journalrepository.org/media/journals/CJAST_ 67/2017/Nov/Singla2442017CJAST37865.pdf.

[9] Ryu, J. H., and Kang, D. 2017. "Physicochemical Properties, Biological Activity, Health Benefits, and 
General Limitations of Aged Black Garlic: A Review.” Molecules 22 (6): 919. Available from: http://www.ncbi.nlm.nih.gov/pubmed/28587168.

[10] Farinde, E. O. 2015. "Chemical and Sensory Properties of Sieved and Unsieved Fortified 'Ogi'." Nature and Science $13 . \quad$ Available from: http://www.sciencepub.net/nature.

[11] Adeniran, H. A. H., and Abiose, S. 2012. "Partial Purification, Characterization and Hydrolytic Activities of Amylases from Bacillus licheniformis and Aspergillus niger Cultured on Agricultural Residues.” African J Biotechnol 11 (6): 1465-77. Available from: http://www.academicjournals.org/AJB/abstracts/abs2012/ 19Jan/Adeniran and Abiose.htm.

[12] Omafuvbe, O. B. 2006. "Effect of Salt on the Fermentation of Soybean (Glycine max) into Daddawa Using Bacillus subtilis as Starter Culture.” African $J$ Biotechnol [Internet]. Academic Journals 5 (10): 1001-5. Available from: http://www.academicjournals.org/journal/AJB/article-abs tract/001072F8762.

[13] Horwitz, W. 2000. AOAC International. Official methods of Analysis of AOAC International. Volume 1, Agricultural Chemicals; Contaminants; Drugs. AOAC International.

[14] Jude-Ojei, B. S., Ajayi, L. A., and Seun, I. O. 2017. "Functional and Pasting Properties of Maize 'Ogi' Supplemented with Fermented Moringa Seeds.” J Food Process Technol 8 (5): 674. Available from: https://www.omicsonline.org/open-access/functional-andpasting-properties-of-maize-ogi-supplemented-withferme nted-moringa-seeds-2157-7110-1000674.pdf.

[15] Mathew, B. K., Adebowale, A. O., and Oladayo, B. O. 2018. "Effect of Traditional and Modified Grain-Soaking Methods on Physicochemical Characteristics and Consumers' Acceptability of Sorghum Ogi." African Journal of Food Science 12 (3): 28-37. Available from: http://www.academicjournals.org/AJFS.

[16] Oluwamukomi, M. O., and Jolayemi, O. S. 2012.
"Physico-Thermal and Pasting Properties of Soy-Melon-Enriched 'Gari' Semolina from Cassava.” Agric Eng Int Cigr J 14 (3): 105-16. Available from: http://pubs.sciepub.com/ajfst/2/5/3/index.html.

[17] Oyarekua, M. A., and Adeyeye, E. I. 2009. “Comparative Evaluation of the Nutritional Quality, Functional Properties and Amino Acid Profile of Co-fermented Maize/Cowpea and Sorghum/Cowpea Ogi as Infant Complementary Food.” Asian J Clin Nutr.

[18] Rojas-Molina, I., Gutiérrez, E., Cortés-Acevedo, M. E., Falcón, A., Bressani, R., Rojas, A., et al. 2008. “Analysis of Quality Protein Changes in Nixtamalized QPM Flours as a Function of the Steeping Time.” Cereal Chem J 85 (3): 409-16. Available from: http://doi.wiley.com/10.1094/CCHEM-85-3-0409.

[19] Ayo-Omogie, H. N., and Ogunsakin, R. 2013. "Assessment of Chemical, Rheological and Sensory Properties of Fermented Maize-Cardaba Banana Complementary Food.” Food Nutr Sci 04 (08): 844-50. Available from: http://www.scirp.org/journal/doi.aspx?DOI=10.4236/fns. 2013.48110.

[20] Bolaji, O. T., Oyewo, A. O., Adepoju, P. A., Bolaji, O. T., and Adepoju, P. A. 2014. "Soaking and Drying Effect on the Functional Properties of Ogi Produce from Some Selected Maize Varieties.” Am J Food Sci Technol 2 (5): 150-7. Available from: http://pubs.sciepub.com/ajfst/2/5/3/index.html.

[21] Lu, D., Sun, X., Yan, F., Wang, X., Xu, R., and Lu, W. 2013. "Effects of High Temperature during Grain Filling Under Control Conditions on the Physicochemical Properties of Waxy Maize Flour.” Carbohydr Polym 98 (1): 302-10. Available from: http://www.ncbi.nlm.nih.gov/pubmed/23987349.

[22] Borode, O. F. 2017. "The Effect of Water and Ethanol Extracts of Ginger and Garlic on the Nutritional Quality and Physico-Chemical Properties of Stored Soymilk." Int J Food Sci Biotechnol 2 (2): 43-50. Available from: http://www.sciencepublishinggroup.com/j/ijfsb. 